\title{
Decreased Cortical Thickness and Local Gyrification in Individuals with Subjective Cognitive Impairment
}

\author{
HyunChul Youn ${ }^{1, *}$, Myungwon Choi ${ }^{2 *}$, Suji Lee ${ }^{3}$, Daegyeom Kim², Sangil Suh ${ }^{4}$, Cheol E. Han ${ }^{2}$, \\ Hyun-Ghang Jeong ${ }^{5,6}$
}

${ }^{1}$ Department of Psychiatry, Soonchunhyang University Bucheon Hospital, Bucheon, ${ }^{2}$ Department of Electronics and Information Engineering, Korea University, Sejong, ${ }^{3}$ Department of Biomedical Sciences, Korea University Graduate School, Departments of ${ }^{4}$ Radiology and ${ }^{5}$ Psychiatry, Korea University Guro Hospital, Korea University College of Medicine, ${ }^{6}$ Korea University Research Institute of Mental Health, Seoul, Korea

\begin{abstract}
Objective: Subjective cognitive impairment $(\mathrm{SCl})$ is associated with future cognitive decline. This study aimed to compare cortical thickness and local gyrification index (LGI) between individuals with $\mathrm{SCl}$ and normal control (NC) subjects. Methods: Forty-seven participants $(27 \mathrm{SCl}$ and $20 \mathrm{NC}$ ) were recruited. All participants underwent brain magnetic resonance imaging scanning and were clinically assessed using the Consortium to Establish a Registry for Alzheimer's Disease (CERAD) battery of tests. We compared cortical thickness and LGl between the two groups and analyzed correlations between cortical thickness/LGI and scores on CERAD protocol subtests in the SCI group for region of interests with significant between-group differences.

Results: Cortical thickness reduction in the left entorhinal, superior temporal, insular, rostral middle frontal, precentral, superior frontal, and supramarginal regions, and right supramarginal, precentral, insular, postcentral, and posterior cingulate regions was observed in the $\mathrm{SCl}$ compared to the $\mathrm{NC}$ group. Cortical thickness in these regions correlated with scores of constructional praxis, word list memory, word list recall, constructional recall, trail making test A, and verbal fluency under the CERAD protocol. Significantly decreased gyrification was observed in the left lingual gyrus of the $\mathrm{SCl}$ group. In addition, gyrification of this region was positively associated with scores of constructional praxis. Conclusion: Our results may provide an additional reference to the notion that $\mathrm{SCl}$ may be associated with future cognitive impairment. This study may help clinicians to assess individuals with $\mathrm{SCl}$ who may progress to mild cognitive impairment and Alzheimer's dementia.
\end{abstract}

KEY WORDS: Alzheimer disease; Cognitive decline; Mild cognitive impairment; Brain cortical thickness; Local gyrification index; Lingual gyrus.

\section{INTRODUCTION}

Subjective cognitive impairment (SCI) is defined by cognitive deficits subjectively perceived by individuals

Received: August 22, 2020 / Revised: October 19, 2020

Accepted: October 26, 2020

Address for correspondence: Cheol E. Han

Department of Electronics and Information Engineering, Korea

University, 2511 Sejong-ro, Sejong 30019, Korea

E-mail: cheolhan@korea.ac.kr

ORCID: https://orcid.org/0000-0002-9994-6861

Hyun-Ghang Jeong

Department of Psychiatry, Korea University Guro Hospital, Korea University College of Medicine, 148 Gurodong-ro, Guro-gu,

Seoul 08308, Korea

E-mail: jeonghg@korea.ac.kr

ORCID: https://orcid.org/0000-0002-0318-5069

*These authors contributed equally to this study as co-first authors. who have normal performance in objective neuropsychological examinations [1]. Evidence that $\mathrm{SCl}$ is associated with an increased risk for future cognitive decline and Alzheimer's disease (AD) dementia is rapidly increasing [1-6].

Many studies have investigated associations between individuals with $\mathrm{SCl}$ and $\mathrm{AD}$-related biomarker abnormalities and have revealed that $\mathrm{SCl}$ is associated with an increased likelihood of biomarker abnormalities consistent with $\mathrm{AD}[3,7,8$ ]. Eliassen et al. [9] and Rami et al. [8] have reported that there was no significant difference between $\mathrm{SCl}$ and mild cognitive impairment $(\mathrm{MCl})$ in terms of cerebrospinal fluid (CSF) $\beta$-amyloid, total tau, and phosphorylated tau pathology. In several neuroimaging studies, greater $\mathrm{SCl}$ severity was related to a greater $\beta$-amyloid

(c) This is an Open-Access article distributed under the terms of the Creative Commons Attribution Non-Commercial License (http://creativecommons.org/licenses/by-nc/4.0) which permits unrestricted non-commercial use, distribution, and reproduction in any medium, provided the original work is properly cited. 
burden [10,11]. Kryscio et al. [12] and Barnes et al. [13] also reported higher levels of neuritic $\beta$-amyloid plaques in post-mortem brain tissue of individuals with SCl. In addition, some studies have conducted fluorodeoxyglucose (FDG)-positron emission tomography (PET) analyses in individuals with SCl. Vannini et al. [14] revealed that increased SCl severity correlated with decreased glucose metabolism in the bilateral precuneus, bilateral inferior parietal lobes, right inferior temporal lobe, right medial frontal gyrus, and right orbitofrontal gyrus. Song et al. [15] also showed periventricular hypometabolism in individuals with $\mathrm{SCl}$ as compared with healthy controls. Moreover, previous structural magnetic resonance imaging (MRI) has shown a reduced volume of the medial temporal lobe, including the hippocampus and the entorhinal cortex in individuals with $\mathrm{SCl}[7,16]$.

Cortical thickness analysis may reveal biomarkers of $\mathrm{SCl}$; cortical thickness is the distance between the white/ gray matter border and the pial surface [17]. Ashburner [18] reported that analyses of cortical thickness could reveal more significant differences than analyses of volume in this disease, which is thought to cause cortical thinning. Cortical thinning has been observed in various conditions, such as normal aging, Huntington's disease, corticobasal degeneration, amyotrophic lateral sclerosis, and schizophrenia [17]. Additionally, in AD pathology, cortical thickness is of great interest $[17,19]$. Previously, measurements of the cortical thickness of individuals with SCI $[9,20,21]$ have been shown to be related to future cognitive decline and AD dementia [1-6]. However, these studies only conducted a region of interest (ROI) analyses. Therefore, in this study, we analyzed the cortical thickness of the whole brain in $\mathrm{SCl}$ with a sub-regional resolution.

Several studies have previously shown that decreased cortical gyrification could occur during the normal aging process [22]. Moreover, cognitive abilities localize to specific regions on the cortical folds of the brain; therefore, folding pattern changes in the brain may also represent the underlying pathology of neurodegenerative disease [23]. Hence, estimation of gyrification may be helpful in assessing the potential for development of neurodegenerative pathologies, such as AD [23]. We considered that this might be particularly important in $\mathrm{SCl}$, as this condition is related to future cognitive decline and $\mathrm{AD}$ dementia [1-6]. The degree of folding of the cortical surface is quantified by the local gyrification index (LGl) [23]. Despite several published studies on brain MRI, only a few studies have assessed cortical folding using the LGI in individuals with SCl.

In this context, we aimed to compare cortical thickness and LGI based on brain MRI between individuals with $\mathrm{SCI}$ and normal control (NC) subjects. Additionally, we analyzed the correlation between cortical thickness/LGl and the score of cognitive measures in the SCI group for the ROls where group differences were significant.

\section{METHODS}

\section{Participants}

Participants were recruited from the psychiatric clinic at the Korea University Guro Hospital and the Guro-gu Center for Dementia. The inclusion criteria for NC subjects were: 1) individuals at least 60 years of age; 2 ) individuals who subjectively reported that there was no abnormality in their cognitive function; and 3) individuals whose scores on all subtests of a neuropsychological battery of tests were within 1.5 standard deviations (SDs) below the mean. The inclusion criteria for $\mathrm{SCl}$ patients were: 1 ) individuals at least 60 years of age; 2 ) individuals who subjectively complained about their cognitive impairment; and 3) individuals whose scores on all subtests of a neuropsychological battery of tests were within $1.5 \mathrm{SDs}$ below the mean. Subjects with neurological impairments (e.g., encephalosclerosis, epilepsy, or traumatic brain injury) or with claustrophobia, substance abuse disorders, and/or with cardiac pacemakers that could affect the results of the MRI scan, were excluded from both NC and $\mathrm{SCl}$ groups in this study. Twenty-seven participants presenting with $\mathrm{SCl}$ and $20 \mathrm{NC}$ participants underwent MRI testing. All participants answered demographic questionnaires and underwent a battery of neuropsychological tests. This study was approved by the Institutional Review Board at Korea University Guro Hospital (KUGH13028). All study subjects provided written informed consent prior to participation. All procedures were performed in accordance with the Helsinki Declaration of 1975, as revised in 2013.

\section{MRI Acquisition}

All participants underwent a structural MRI examination at the Brain Imaging Center of Korea University. The 
scan parameters were: $b=1,000 \mathrm{~s} / \mathrm{mm}^{2}$, echo time (TE) / repetition time $(\mathrm{TR})=84 \mathrm{~ms} / 6.3 \mathrm{~s}$; matrix $=128 \times 128$ on a $230 \times 230 \mathrm{~mm}$ field-of-view; 3-mm slices without a gap, resulting in voxels of $1.8 \times 1.8 \times 3.0 \mathrm{~mm}$. Sufficient signal-to-noise ratios were provided by four magnitude averages. Volumetric T1-weighted anatomic images were acquired using a magnetization-prepared rapid gradientecho sequence (TE/TR/inversion time $[\mathrm{TI}]=2.60 \mathrm{~ms} / 1.9$ $\mathrm{s} / 900 \mathrm{~ms} ; 256 \times 256 \times 176$ matrix for $0.86 \times 0.86 \times 1 \mathrm{~mm}$ voxels).

\section{Image Processing and Cortical Measurements}

We computed cortical thickness and LGI from T1weighted MR images using FreeSurfer version 5.1.0 (Massachusetts General Hospital, Harvard Medical School; http://surfer.nmr.mgh.harvard.edu). This software extracts various surfaces, including the pial surface (the outer boundary of the gray matter), and white surfaces (the boundary between the gray and white matter) [24,25]. We processed T1-weighted MR images of each subject using the software following the recommended reconstruction procedure including visual validation and correction of the intermediate results by neurophysiologists. The extracted surfaces consist of vertices and are isomorphic; they have the identical number of vertices with identical relations between vertices. The cortical thickness was estimated by the closest distance between two surfaces at each vertex of the white surface [17].

The LGI is defined as a metric that quantifies the amount of cortex buried within the sulcal folds, as compared to the amount of visible cortex. In this study, we used FreeSurfer's built-in function to determine this value $[26,27]$. A larger LGI indicates a more folded area, while a smaller LGI represents a smoother area. In order to compute LGI, first, a smooth outer surface that wraps the pial surface is created. Then, a circular ROI is created, centered on a particular vertex and the pial surface. The LGI value was computed as the ratio between the areas of those two ROls, considering the distance between the two surfaces. Repeating this procedure over the vertices of the pial surface generated an LGI map.

Inter-subject correspondence of data-points is critical in surface analysis. Due to inter-subject variability in brain shape, the number of vertices on the brain surfaces often varies across subjects, and this may seriously hamper analysis. FreeSurfer provides spatial registration of the surfaces between subjects, using the major folding patterns, ensuring isomorphic surfaces with an identical number of vertices in all subjects [28]. We registered each subject's surfaces on the FreeSurfer's average subject, and resampled cortical thickness and LGI. Then, we smoothed the resampled values with a full-width at half-maximum filter with a radius of $10 \mathrm{~mm}$.

\section{Demographic and Cognitive Measures}

For all participants, information about age, sex, and education level were collected. Additionally, the apolipoprotein $\mathrm{E}$ (APOE) genotype was also determined, given that APOE alleles are accepted genetic determinants of $\mathrm{AD}$ risk [29].

The cognitive functions of participants were measured using the Consortium to Establish a Registry for AD (CERAD) neuropsychological battery of clinical testing [30]. In this study, we used the Korean version of CERAD and the Korean specific age-, sex-, and education-adjusted norms [31]. All participants scored within 1.5 SD below the mean. The Mini-Mental State Examination (MMSE) is an easy to use a measure of cognitive function that has been widely implemented in the screening and clinical evaluation of patients with dementia [32]. We conducted the MMSE in the Korean version of the CERAD assessment package and adopted the Korean specific age-, sex-, and education-adjusted norms base on z-score [33]. In addition, we included the Korean version of the Geriatric Depression Scale (GDS) to assess the degree of distress in our subjects [34].

\section{Statistical Analysis}

Differences between the $\mathrm{SCl}$ and the $\mathrm{NC}$ groups in demographic and cognitive measures were analyzed using Predictive Analytics Software 18.0 for Windows (IBM Co., Armonk, NY, USA; i.e., Mann - Whitney test for continuous variables and the $\chi^{2}$ test or Fisher's exact test for categorical variables).

We compared the cortical thickness and LGI at the level of vertices between subjects in the $\mathrm{SCl}$ and $\mathrm{NC}$ group, using the general linear model (GLM) with the Query, Design, Estimate, Contrast interface of FreeSurfer (https://surfer.nmr.mgh.harvard.edu/fswiki/Qdec). Specifically, we tested the group differences in cortical measures between $\mathrm{SCl}$ and $\mathrm{NC}$ subjects, controlling for the effects of age, sex, and education levels by using the 
Table 1. Basic characteristics and cognitive measures of normal control and subjective cognitive impairment groups

\begin{tabular}{|c|c|c|c|}
\hline Variable & $N C(n=20)$ & $\mathrm{SCl}(\mathrm{n}=27)$ & $p$ value $^{\mathrm{a}}$ \\
\hline Age (yr) & $70.20 \pm 7.46$ & $69.07 \pm 8.14$ & 0.511 \\
\hline Sex & & & 0.188 \\
\hline Male & $8(40.0)$ & $6(22.2)$ & \\
\hline Female & $12(60.0)$ & $21(77.8)$ & \\
\hline Education (yr) & $10.58 \pm 5.39$ & $5.93 \pm 5.25$ & $0.005^{* *}$ \\
\hline APOE4 allele carriers & $5(25.0)$ & $8(29.6)$ & 0.831 \\
\hline MMSE & $27.05 \pm 2.88$ & $23.67 \pm 4.29$ & $0.002^{* *}$ \\
\hline GDS & $8.44 \pm 5.54$ & $16.96 \pm 6.20$ & $<0.001^{* *}$ \\
\hline \multicolumn{4}{|l|}{ CERAD } \\
\hline Constructional praxis & $9.94 \pm 1.95$ & $8.96 \pm 1.59$ & $0.011^{*}$ \\
\hline Word list memory & $17.06 \pm 5.05$ & $15.54 \pm 4.22$ & 0.131 \\
\hline Word list recall & $6.63 \pm 1.26$ & $5.31 \pm 2.02$ & $0.026^{*}$ \\
\hline Word list recognition & $9.47 \pm 0.70$ & $8.96 \pm 1.11$ & 0.140 \\
\hline Constructional recall & $8.00 \pm 2.20$ & $5.38 \pm 2.98$ & $0.004^{* *}$ \\
\hline Verbal fluency & $15.79 \pm 3.85$ & $13.42 \pm 3.29$ & 0.052 \\
\hline Boston Naming Test & $12.84 \pm 2.04$ & $10.42 \pm 2.64$ & $0.001^{* *}$ \\
\hline Trail Making Test A (sec) & $58.57 \pm 14.99$ & $91.08 \pm 49.43$ & $0.046^{*}$ \\
\hline
\end{tabular}

Values are presented as mean \pm standard deviation or number $(\%)$.

$\mathrm{NC}$, normal control; $\mathrm{SCl}$, subjective cognitive impairment; $\mathrm{APOE}$, apolipoprotein E; MMSE, Mini-Mental State Examination; GDS, Geriatric Depression Scale; CERAD, Consortium to Establish a Registry for Alzheimer's Disease.

a $p$ value were calculated using the chi-squared test or Fisher's exact test and the Mann - Whitney test.

${ }^{*} p<0.05 .{ }^{* *} p<0.01$.

following GLM equation. We assumed different offsets and the same slope across groups at each vertex i:

$y_{i}=b_{0}+b_{1} \cdot$ group $+b_{2} \cdot$ age $+b_{3} \cdot \operatorname{sex}+b_{4} \cdot$ education level

where $y_{i}$ is the cortical measure of the $i$-th vertex (cortical thickness or $\mathrm{LGI}$ ), and $b_{0}, b_{1}, b_{2}, b_{3}$ and $b_{4}$ correspond to the regression coefficients for each term: bias, group, age, sex, and education level. The resulting significance map yielded $\mathrm{a}-\log _{10}(p$ value) and was thresholded with 2.3 for cortical thickness and 2.0 for LGI (2.3 and 2.0 corresponds to $p=0.005$ and $p=0.01$, respectively) for visualization. We reported clusters with an area larger than $50 \mathrm{~mm}^{2}$ and with a group difference of mean cortical thickness (uncorrected $p<0.01$ ). The adjusted cortical measures in figures were computed by deducting the confounder terms from both sides of the GLM equation.

We evaluated the correlation between the measurements and scores obtained from the CERAD battery of tests in the $\mathrm{SCl}$ group for regions where the betweengroup differences were significant. We first computed the average values of cortical thickness and the LGI for the identified cluster. We employed a partial correlation coefficient between the average values and CERAD scores, controlling for age, sex, and education levels. We used our in-house MATLAB codes (MathWorks, Natick, MA, USA) for all additional analyses and parameter visualization.

\section{RESULTS}

Table 1 shows the basic characteristics and cognitive measures for both groups. The mean ages in the $\mathrm{NC}$ and $\mathrm{SCl}$ groups were 70.20 years (SD $=7.46$ years) and 69.07 years ( $\mathrm{SD}=8.14$ years), respectively. More females than males participated in both groups $(\mathrm{NC}, 60.0 \%$; SCl, $77.8 \%)$. The SCI participants were less educated than the NC participants. Naturally, the two groups differed from each other in cognitive measure scores, including MMSE and CERAD subtests. GDS scores were also different between groups.

\section{Group Comparisons between the $\mathrm{SCl}$ and NC Groups}

Our analyses showed significant between-group differences in both cortical thickness and LGI scores (Table 2). The $\mathrm{SCl}$ subjects showed significantly reduced mean cortical thickness in the left entorhinal, superior temporal, insula, rostral middle frontal, precentral, superior frontal, and supramarginal regions, and the right supramarginal, precentral, insula, postcentral (uncorrected $p$ equals to or 
Table 2. Cluster regions with significant group differences in cortical thickness and local gyrification index

\begin{tabular}{|c|c|c|c|c|c|c|c|}
\hline \multirow{3}{*}{$\begin{array}{c}\mathrm{SCl}<\mathrm{NC} \\
\text { Cluster }\end{array}$} & \multicolumn{4}{|c|}{ Peak location ${ }^{\mathrm{a}}$} & \multicolumn{3}{|c|}{ Overall } \\
\hline & \multirow{2}{*}{$P_{\text {peak }}^{b}$} & \multicolumn{3}{|c|}{ Talairach coordinate } & \multirow{2}{*}{ Area $^{c}$} & \multirow{2}{*}{$N_{\text {vertices }}{ }^{d}$} & \multirow{2}{*}{ F-statistics ${ }^{e}$} \\
\hline & & $x$ & Y & Z & & & \\
\hline \multicolumn{8}{|l|}{ Cortical thickness } \\
\hline L entorhinal & $<0.001$ & -24.8 & -7.2 & -28.5 & 281.18 & 635 & $24.251^{*}$ \\
\hline L superior temporal & 0.001 & -62.6 & -17.3 & 0.4 & 151.09 & 395 & $18.792 *$ \\
\hline L superior temporal & 0.001 & -52.5 & -1.8 & -6.1 & 119.63 & 228 & $15.193^{*}$ \\
\hline L insula & $<0.001$ & -31.7 & 8.3 & 11.4 & 94.63 & 305 & $13.802^{* *}$ \\
\hline L rostral middle frontal & $<0.001$ & -22.5 & 44.0 & 16.3 & 70.23 & 109 & $12.159^{* *}$ \\
\hline L precentral & 0.001 & -56.7 & -1.0 & 10.1 & 68.03 & 167 & $12.654^{* *}$ \\
\hline L superior frontal & 0.001 & -22.4 & 4.0 & 43.6 & 58.21 & 142 & $14.415^{*}$ \\
\hline L supramarginal & 0.001 & -49.5 & -30.3 & 34.8 & 56.09 & 136 & $12.198^{* *}$ \\
\hline L superior frontal & 0.001 & -10.7 & 21.9 & 34 & 55.4 & 127 & $12.668^{* *}$ \\
\hline R supramarginal & $<0.001$ & 57.8 & -38.1 & 30.1 & 238.91 & 464 & $20.106^{*}$ \\
\hline R precentral & $<0.001$ & 36.0 & -10.7 & 59.8 & 208.54 & 475 & $20.618^{*}$ \\
\hline R supramarginal & $<0.001$ & 58.9 & -34.3 & 18.1 & 153.12 & 339 & $16.537^{*}$ \\
\hline R supramarginal & 0.001 & 55.3 & -24.3 & 26.7 & 96.39 & 235 & $17.409^{*}$ \\
\hline$R$ insula & $<0.001$ & 34.1 & -22.1 & 20.1 & 79.76 & 269 & $15.174 *$ \\
\hline R postcentral & 0.001 & 33.8 & -30.3 & 61.2 & 71.84 & 182 & $14.320^{*}$ \\
\hline R precentral & $<0.001$ & 40.2 & -0.4 & 15.9 & 71.53 & 221 & $11.871^{* *}$ \\
\hline $\mathrm{R}$ posterior cingulate & 0.002 & 6.4 & -23.4 & 38.1 & 52.36 & 120 & $11.522^{* * *}$ \\
\hline \multicolumn{8}{|l|}{ LGI } \\
\hline L lingual & 0.009 & -17.9 & -78.9 & -3.4 & 12.70 & 14 & $7.452^{* * * *}$ \\
\hline
\end{tabular}

$\mathrm{SCl}$, subjective cognitive impairment; $\mathrm{NC}$, normal control; LGI, local gyrification index.

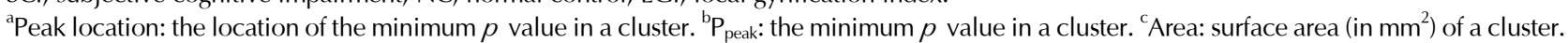
${ }^{d} \mathrm{~N}_{\text {vertices: }}$ : the number of vertices in a cluster. ${ }^{\mathrm{e}} \mathrm{F}$-statistics: $\mathrm{F}$ statistics for group comparison of mean cortical thickness between the $\mathrm{NC}$ and $\mathrm{SCl}$ groups, controlling for the effects of age, sex, and education level, and the relevant $p$ value.

${ }^{*} p<0.001 .{ }^{* *} p=0.001 .{ }^{* * *} p=0.002 .{ }^{* * * *} p=0.009$.

below 0.001 for the results until here), and posterior cingulate regions (uncorrected $p=0.002$ ), as compared to the NC group (Figs. 1A, 1B, 2). The left lingual gyrus had a reduced LGI in $\mathrm{SCl}$ subjects (uncorrected $p=0.009$ ), although the area of its identified cluster did not exceed 50 $\mathrm{mm}^{2}$ (Figs. 1C, 2).

\section{Correlation of Cortical Thickness and LGI Values with Cognitive Scores}

Cortical thickness of regions that differed significantly between groups showed positive correlations with $\mathrm{SCI}$ group scores of constructional praxis, word list memory, word list recall, constructional recall, and verbal fluency under the CERAD battery of cognitive testing. Negative correlations were found for the time taken for the trail making test A (shown in seconds, Table 3). The LGl of the left lingual gyrus was positively associated with scores from the constructional praxis subtest (Table 3, Fig. 3; Supplementary Figs. $1-4$, available online).

\section{DISCUSSION}

In the present study, we investigated the differences between MRI brain scans of subjects with $\mathrm{SCl}$ and NC participants, focusing on cortical thickness and LGl values. Cortical thickness was reduced in $\mathrm{SCl}$ subjects as compared to that in the NC group, specifically in the left entorhinal, superior temporal, insula, rostral middle frontal, precentral, superior frontal, and supramarginal regions, and the right supramarginal, precentral, insula, postcentral, and posterior cingulate regions. Analysis of the LGl revealed significantly decreased gyrification in the left lingual gyrus in $\mathrm{SCl}$ subjects. In addition, we demonstrated the association of scores in the subtests in the CERAD battery in the SCl group with LGI and cortical thickness.

Changes in the brain's cortical folding geometry have been associated with cognitive decline [35], but there have been few studies that have analyzed cortical gyrification in individuals with SCI. Liu et al. [36] estimated 


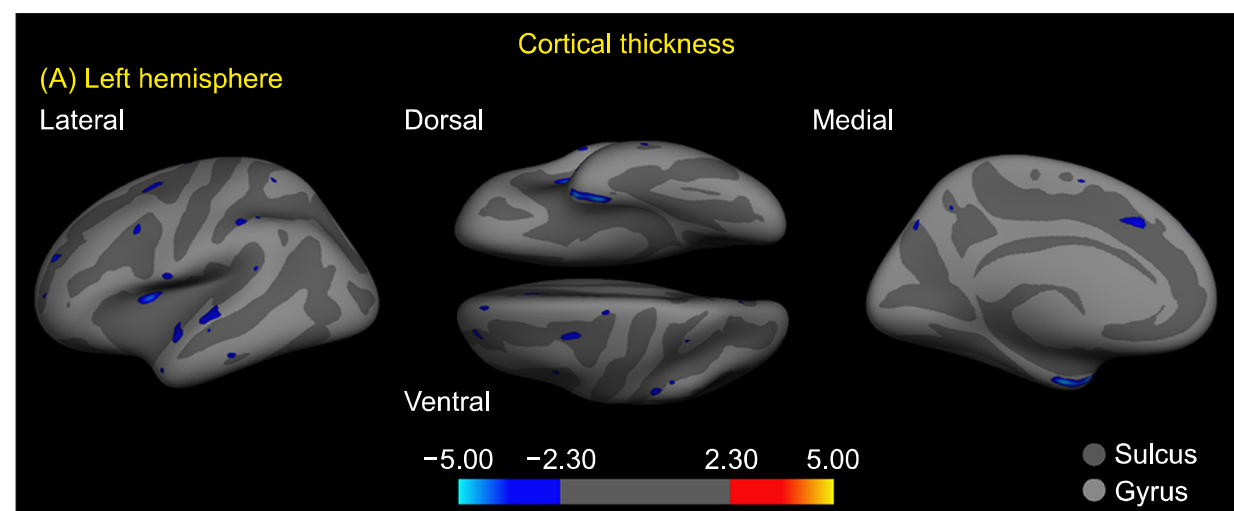

(B) Right hemisphere

Lateral
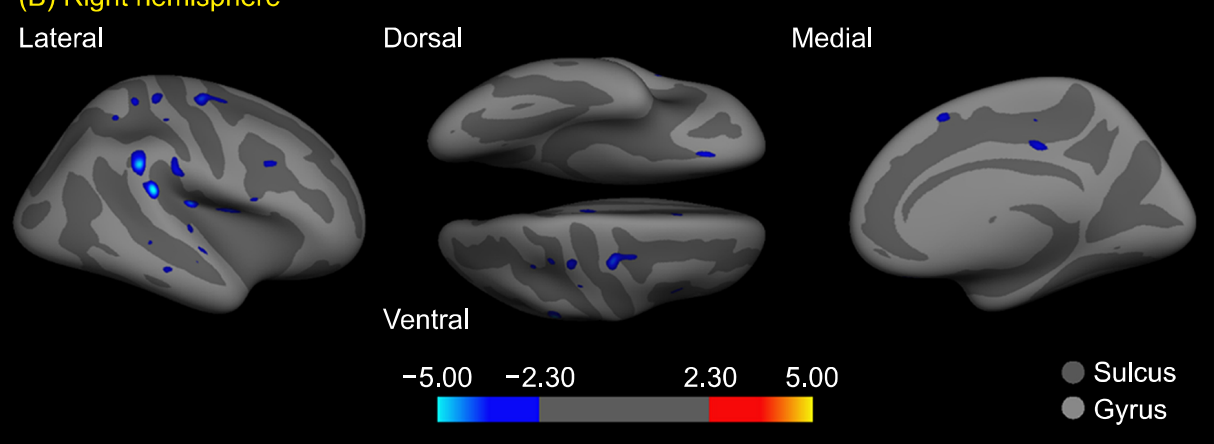

(C) Left hemisphere

Lateral

Local gyrification index

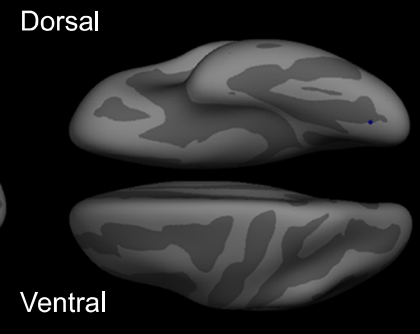

Medial

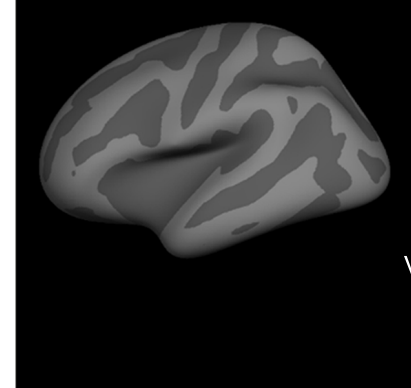

$-5.00$

$-2.00$

2.00

5.00

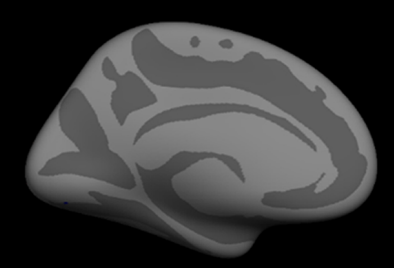

Fig. 1. Whole-brain significance map of cortical thickness and local gyrification index (LGI) differences between the subjective cognitive impairment $(\mathrm{SCl})$ and normal control (NC) group. Each inflated cortical surface shows clusters of regions with cortical thickness differences between the groups in the (A) left hemisphere, in the (B) right hemisphere, and (C) between-group LGl differences in the left hemisphere. The maps consist of $-\log _{10}$ ( $p$ value) where 2.3 corresponds to $p=0.005$ for cortical thickness, and 2.0 corresponds to $p=0.01$ for the LGl. The cooler (bluer) color indicates decreased values in the SCI group compared to the NC group, while the warmer (redder) color indicates increased values (not found). The only clusters with areas exceeding $50 \mathrm{~mm}^{2}$ are shown in (A, B).

the global gyrification index values in early-stage AD and revealed negative associations with the severity of $\mathrm{AD}$. They also reported positive associations between the global gyrification index values and MMSE scores. Lebed et al. [23] used LGl scores on individuals with a CDR score of $0,0.5$, and 1 , and revealed various regions that showed decreased gyrification as CDR scores increased.
These studies demonstrated the value of cortical gyrification analysis in the early stages of AD $[23,36]$.

Our study used LGI analysis to investigate cortical gyrification in individuals with $\mathrm{SCl}$ and identified a gyrification decrease in the lingual gyrus of the brain, although the cluster regions found were relatively small. Previous studies have reported that the lingual gyrus plays 

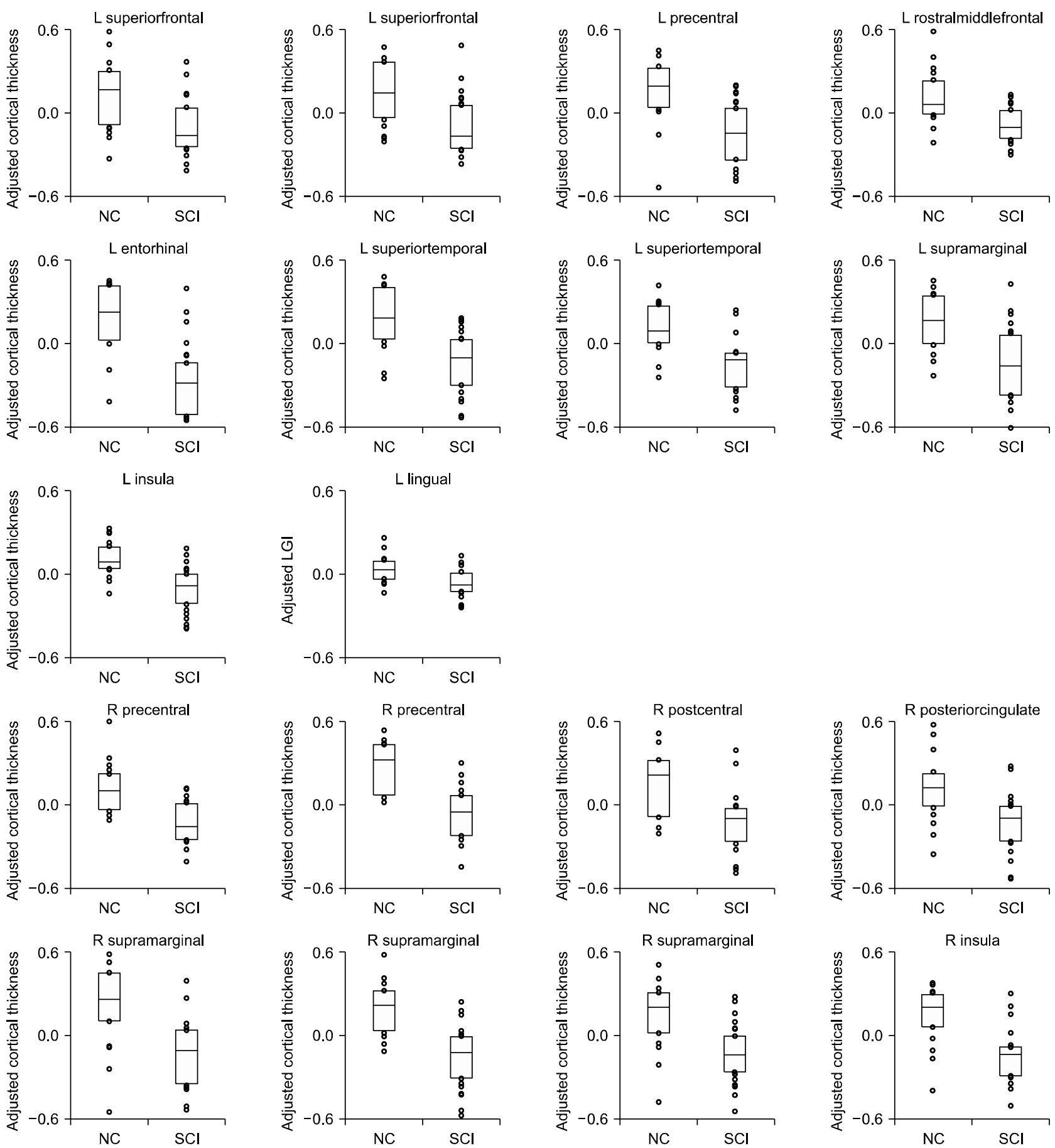

Fig. 2. Group comparison of adjusted mean cortical thickness and local gyrification index between subjective cognitive impairment (SCI) and normal control (NC) groups. Each subplot corresponds to its respective cluster, shown in Table 2. The adjusted values contained only the group effect; we first averaged the cortical measurement over an identified cluster, and controlled for the effects of age, sex, and education level. The boxes represent the first and third quartiles of the data with the median (solid line), whereas the circles outside the boxes represent data-points outside the central quartiles.

a critical role in mediating cognitive deficits in visual processing and memory $[37,38]$, which have been associated with $A D$ [39]. Our results are noteworthy because
$\mathrm{SCl}$ is known to be associated with an increased risk of developing AD dementia [1-6]. The positive correlation between the lingual gyrus and constructional praxis tasks 
Table 3. Correlation between cortical thickness/local gyrification index and cognitive measures in the subjective cognitive impairment group

\begin{tabular}{|c|c|c|c|}
\hline Cognitive measures & Cluster & $R^{\mathrm{a}}$ & $p$ value $^{\mathrm{b}}$ \\
\hline \multicolumn{4}{|l|}{ Cortical thickness } \\
\hline \multirow[t]{7}{*}{ Constructional praxis } & Left superior temporal & 0.456 & 0.019 \\
\hline & Left insula & 0.547 & $0.004^{*}$ \\
\hline & Left superior frontal & 0.535 & $0.005^{*}$ \\
\hline & Left supramarginal & 0.681 & $<0.001^{*}$ \\
\hline & Right supramarginal & 0.409 & 0.038 \\
\hline & Right insula & 0.533 & $0.005^{*}$ \\
\hline & Right precentral & 0.443 & 0.024 \\
\hline \multirow[t]{6}{*}{ Word list memory } & Left superior temporal & 0.648 & $<0.001^{*}$ \\
\hline & Left superior temporal & 0.490 & 0.011 \\
\hline & Left superior frontal & 0.560 & $0.003^{*}$ \\
\hline & Right insula & 0.407 & 0.039 \\
\hline & Right precentral & 0.496 & 0.010 \\
\hline & Right posterior cingulate & 0.499 & 0.010 \\
\hline \multirow[t]{7}{*}{ Word list recall } & Left superior temporal & 0.455 & 0.020 \\
\hline & Left precentral & 0.390 & 0.049 \\
\hline & Left superior frontal & 0.660 & $<0.001^{*}$ \\
\hline & Right supramarginal & 0.515 & 0.007 \\
\hline & Right insula & 0.401 & 0.042 \\
\hline & Right precentral & 0.523 & $0.006^{*}$ \\
\hline & Right posterior cingulate & 0.721 & $<0.001^{*}$ \\
\hline \multirow[t]{3}{*}{ Constructional recall } & Left rostral middle frontal & -0.402 & 0.042 \\
\hline & Left supramarginal & -0.519 & 0.007 \\
\hline & Right supramarginal & 0.476 & 0.014 \\
\hline \multirow[t]{2}{*}{ Verbal fluency } & Right supramarginal & 0.459 & 0.018 \\
\hline & Right postcentral & 0.398 & 0.044 \\
\hline \multirow[t]{7}{*}{ Trail making test A (sec) } & Left superior temporal & -0.738 & $<0.001^{*}$ \\
\hline & Left insula & -0.657 & $<0.001^{*}$ \\
\hline & Left supramarginal & -0.605 & $0.001^{*}$ \\
\hline & Right supramarginal & -0.445 & 0.026 \\
\hline & Right supramarginal & -0.428 & 0.033 \\
\hline & Right insula & -0.413 & 0.040 \\
\hline & Right posterior cingulate & -0.540 & $0.005^{*}$ \\
\hline \multicolumn{4}{|c|}{ 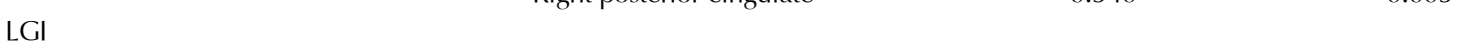 } \\
\hline Constructional praxis & Left lingual & 0.549 & $0.004^{*}$ \\
\hline
\end{tabular}

LGI, local gyrification index.

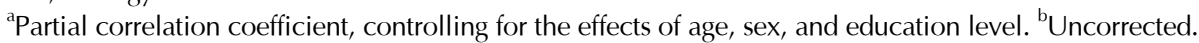

*Bonferroni corrected $p<0.05$.

was also consistent with the role of the lingual gyrus in cognitive processing $[37,38]$. In addition, Jeong et al. [40] have found reduced cerebral glucose metabolism in the lingual gyrus of older women with subjective memory impairment. Other studies have found that individuals with $\mathrm{MCl}$ presented gray matter volume reduction in the lingual gyrus [41,42]. Yetkin et al. [43] also detected deactivation of the lingual gyrus in an $\mathrm{MCl}$ group, based on functional MRI analysis. Our study reproduced similar findings in the context of gyrification changes in subjects with $\mathrm{SCl}$.

Cortical thinning of the entorhinal and posterior cingu- late regions was found in individuals presenting SCl. These results were plausible, given that these regions have been associated with memory dysfunction, which is at the core of functional disability in AD $[44,45]$. Previous studies have also reported the association between the entorhinal/posterior cingulate regions and cognitive impairment. Some studies of SCl individuals have shown cortical thickness reduction in these regions $[9,20,21]$. Ryu et al. [16] showed lower entorhinal cortical volumes in individuals with subjective memory impairment, and Jeong et al. [40] found reduced glucose metabolism in the posterior cingulate of elderly women with subjective memory 

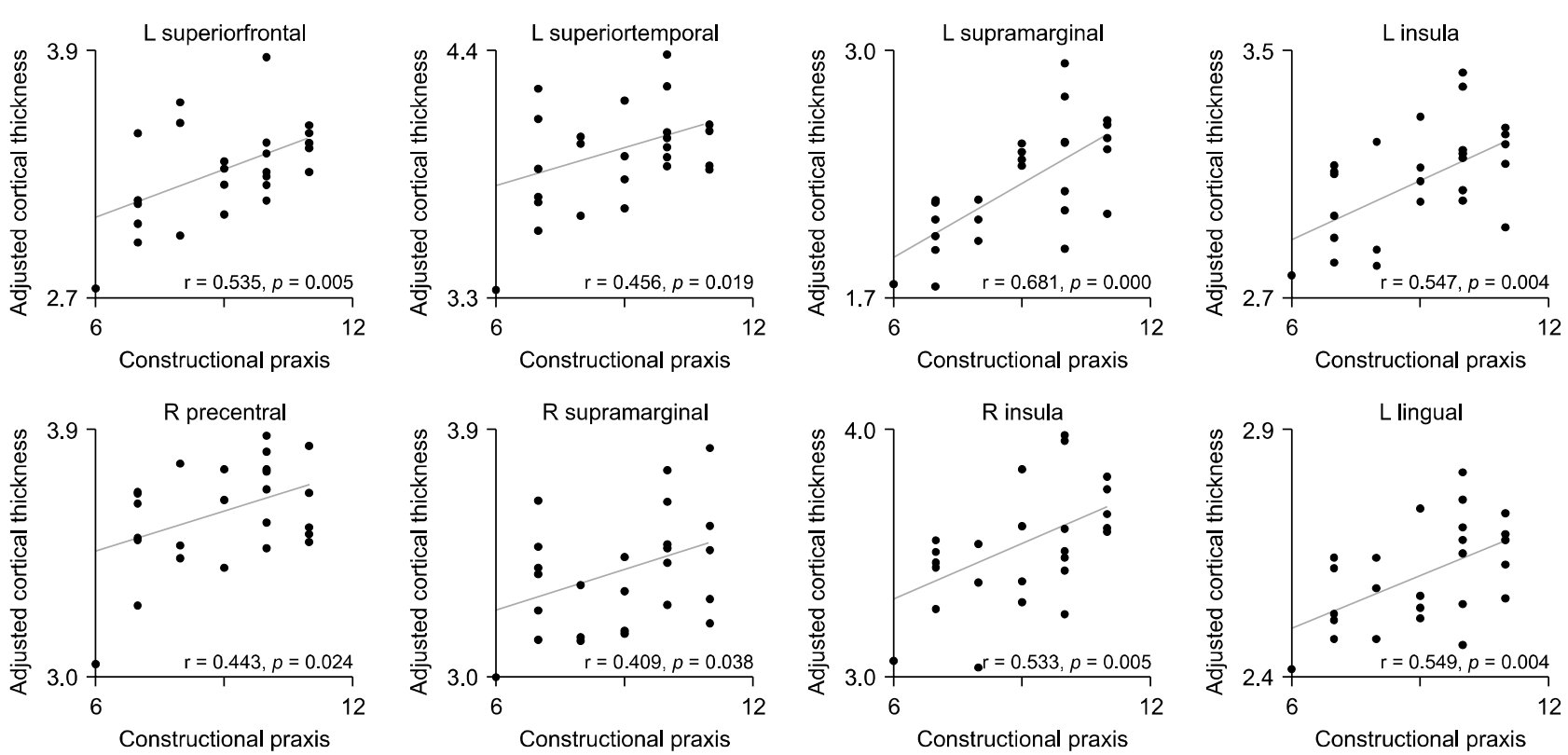

Fig. 3. Correlation of constructional praxis, in the Consortium to Establish a Registry for Alzheimer's Disease (CERAD) assessment, with the adjusted mean cortical thickness and local gyrification index (LGI). Each subplot corresponds to a cluster in Table 3. We used partial correlation coefficients between the CERAD score and the average values of cortical measurements, controlling for age, sex, and education level using a general linear model, where the average value was obtained over each cluster.

impairment. In addition, Liu et al. [46] reported cortical thinning of both these regions in progressive $\mathrm{MCl}$ individuals, as compared with control groups. Our correlation analysis showed the association between the posterior cingulate region and word list memory/recall tasks performed by individuals in the $\mathrm{SCl}$ group. This is also consistent with the functional role of the posterior cingulate region.

The superior frontal and rostral middle frontal regions also showed a decrease in cortical thickness in individuals in the $\mathrm{SCl}$ group. These regions are known to play a critical role in executive and higher cognitive functions such as working memory $[47,48]$. Consistent with these roles, thinning in the superior frontal region was found to be associated with word list memory tasks in our correlation analysis. Our findings on the superior frontal and rostral middle frontal regions were consistent with those of previous studies. Meiberth et al. [21] reported cortical thinning of the superior frontal region in individuals with $\mathrm{SCl}$. Structural abnormalities in the superior frontal region were also found in familial studies on AD mutation carriers and prodromal AD $[49,50]$. In addition, Liu et al. [46] reported cortical thinning of the superior frontal and rostral middle frontal regions in an $\mathrm{AD}$ group.

Additionally, a reduction in the cortical thickness in in- dividuals in the $\mathrm{SCl}$ group was also observed in the supramarginal and superior temporal regions. These regions were positively correlated with various cognitive domains in the CERAD battery of tests performed by subjects with $\mathrm{SCl}$. The supramarginal and superior temporal regions are related to dysfunction of visual and auditory word recognition, respectively, which is also associated with $A D$ $[39,51]$. Cerebral glucose hypometabolism in the superior temporal region was observed in studies on elderly women with subjective memory impairment [40]. Blanc et al. [50] previously reported gray matter atrophy of the superior temporal region in individuals with prodromal AD. Other studies have found cortical thinning of the supramarginal region in patients with early-stage $A D$ and familial AD mutation carriers $[49,52]$. In addition, Lau et al. [53] conducted a meta-analysis based on resting-state functional MRI, and reported abnormalities in the supramarginal region of subjects with $\mathrm{MCl}$ as compared to healthy controls. Additionally, Redolfi et al. [54] demonstrated changes in the supramarginal and superior temporal regions in individuals with $A D$, based on structural MRI scans. Our results were consistent with these previous findings.

The insula plays a major role in speech and language processing [55]. Patients with AD frequently show lan- 
guage difficulties [39]; therefore, thinning of the insular cortex in individuals with $\mathrm{SCl}$ was plausible. The results of previous studies were also consistent with those of our study. Davatzikos et al. [56] previously reported a decrease in the volume of the insular region in $\mathrm{MCl}$ individuals who later developed AD. Eskildsen et al. [57] analyzed the cortical thickness of subjects with progressive $\mathrm{MCl}$, and found cortical thinning in the insular region.

In addition to the abovementioned regions, cortical thinning was also found in precentral and postcentral regions of the brain. These regions are associated with motor and somatosensory functions, respectively $[58,59]$. Song et al. [15] showed precentral glucose hypometabolism in these regions in an $\mathrm{MCl}$ group, and Liu et al. [46] reported cortical thinning of these regions in a group of AD patients, as compared to NCs. Nonetheless, there have been few studies explaining the association between these regions and AD pathology. Further studies are needed to clarify the details of these associations.

Previous studies have reported cortical thinning in the entorhinal, fusiform, posterior cingulate, parahippocampal, and inferior parietal cortices $[9,20,21]$. Our findings on cortical thickness overlapped with these previous results in part, perhaps due to the differences in spatial resolution used in analysis between those studies and our own. In previous studies, ROI analyses were conducted, rather than the sub-regional whole-brain analyses that we used in our study. In this respect, our analyses on cortical thickness deserve attention. In addition, as far as we know, no previous study had included an LGI analysis of brain MRI scans of individuals with $\mathrm{SCl}$. Furthermore, our results on the LGI values were compatible with previous reports on AD pathology. Our findings on both cortical thickness and LGI scores may support those of previous studies that found association between $\mathrm{SCl}$ and future cognitive impairment [1-6]. Further longitudinal studies, examining earlier to later phases of $A D$, are needed to clarify and validate these findings.

Nonetheless, there are some limitations to this study. First, the sample size of our study was relatively small. This may reduce the generalizability of the present results. Second, we only compared participants with $\mathrm{SCl}$ and $\mathrm{NC}$ subjects. Considering that $\mathrm{SCl}$ is associated with an increased risk of future cognitive decline and $\mathrm{AD}$ dementia [1-6], a study that includes both patients with $\mathrm{MCl}$ and those with AD would be more appropriate and useful. In addition, a longitudinal design that can observe changes in cognitive function and brain MRI may be more informative than a cross-sectional design as used in this study. Third, we did not check the cognitive domain of main complaints in our SCl participants. Classifying and analyzing the groups according to their main cognitive complaints will help to advance the findings of this study. Fourth, this study did not control confounding factors especially depressive symptom (GDS score) - that can be associated with SCI. However, given that many older adults who have cognitive complaints and visit the psychiatric clinic tend to have depressive symptoms - whether major or minor degree - , relatively higher GDS scores may reflect this real-life situation in the clinical setting [60]. Further studies controlling various confounding factors may be necessary to verify and advance our findings. Fifth, the score of constructional recall was negatively associated with the left rostral middle frontal and left supramarginal regions in our study. These results were unexpected and cannot be explained in the context of the major functions of these regions and the results of previous studies. Sixth, the size of the identified regions in our analysis was relatively small. Thus, the results should be interpreted with caution; they might contain false positives, especially given the small patient sample size. Despite these caveats, our results are indeed compatible with those of previous studies and the major functional roles of the ROls. Our identified brain regions were confirmed to demonstrate significant differences between the two groups based on the average value of cortical measurement over the identified brain regions (Figs. 2, 3). Moreover, we found a significant association between cognitive decline in $\mathrm{SCl}$ and the average value of cortical measurements over the identified brain regions (Fig. 3; Supplementary Figs. 1-4, available online). In addition, unlike previous studies, this study used LGI analysis in individuals with $\mathrm{SCl}$. Though the very local difference in LGI of the lingual gyrus, it was first reported, and was strongly correlated with the specific cognitive function. Further studies are needed to clarify the regions that represent reduced gyrification in individuals with SCI. Finally, the absence of additional biomarkers indicating AD pathology, such as CSF biomarkers, FDG, or amyloid PET-imaging, is another limitation of this study. The addition of AD biomarkers may increase reliability and can help to max- 
imize the findings of this study.

This study compared structural MRI of $\mathrm{SCl}$ individuals with those of a NC group by analyzing brain cortical thickness and local gyrification. In the $\mathrm{SCl}$ group, we found cortical thinning in the left entorhinal, superior temporal, insula, rostral middle frontal, precentral, superior frontal, and supramarginal regions, and the right supramarginal, precentral, insula, postcentral, and posterior cingulate regions, as compared to the NC group. In addition, decreased gyrification was found in the left lingual gyrus of $\mathrm{SCl}$ individuals.

In conclusion, our results may provide a further basis for the concept that SCI may be associated with future cognitive impairment. This study may help clinicians assess individuals with $\mathrm{SCl}$ who may progress to $\mathrm{MCl}$ and $A D$, and eventually, may contribute to early diagnosis of AD. In addition, our LGI analyses in individuals with $\mathrm{SCl}$ may form a basis for future studies.

\section{- Acknowledgments}

This work was supported by the Korea Health Technology R\&D Project through the Korea Health Industry Development Institute (KHIDI) that was funded by the Ministry of Health \& Welfare, Republic of Korea (HC15C1509 to HG Jeong and HI19C0645 to HG Jeong \& CE Han); the Basic Science Research Program through the National Research Foundation of Korea (NRF), funded by the Ministry of Science and ICT (NRF-2015R1C1A1A0 1052172 to HG Jeong). The funders had no role in study design, data collection and analysis, decision to publish, or preparation of the manuscript.

We thank the participants in this study. The authors also wish to thank Seulki Kim for assistance in data collection.

\section{Conflicts of Interest}

No potential conflict of interest relevant to this article was reported.

\section{Author Contributions}

Data Curation: Hyun-Ghang Jeong, Suji Lee, HyunChul Youn. Formal Analysis: Myungwon Choi, Daegyeom Kim, Cheol E. Han, HyunChul Youn, Sangil Suh. Funding Acquisition: Hyun-Ghang Jeong, Cheol E. Han. Investigation: Sangil Suh, Hyun-Ghang Jeong, Suji Lee. Methodology: Myungwon Choi, Cheol E. Han. Project Administration: Hyun-Ghang Jeong. Resources: Hyun-Ghang Jeong,
Sangil Suh. Software: Myungwon Choi, Daegyeom Kim, Cheol E. Han. Supervision: Hyun-Ghang Jeong. Visualization: Myungwon Choi, Daegyeom Kim, Cheol E. Han. Writing-Original Draft Preparation: HyunChul Youn, Myungwon Choi. Writing-Review \& Editing: HyunChul Youn, Myungwon Choi, Cheol E. Han, Hyun-Ghang Jeong.

\section{ORCID}

HyunChul Youn

https://orcid.org/0000-0002-6557-5628

Myungwon Choi

Suji Lee https://orcid.org/0000-0002-8320-7300

Daegyeom Kim https://orcid.org/0000-0002-5016-7680

Sangil Suh

Cheol E. Han https://orcid.org/0000-0002-9994-6861 https://orcid.org/0000-0002-2234-2955 https://orcid.org/0000-0001-8933-0492

Hyun-Ghang Jeong https://orcid.org/0000-0002-0318-5069

\section{REFERENCES}

1. Jessen $\mathrm{F}$, Amariglio RE, van Boxtel M, Breteler M, Ceccaldi M, Chételat $\mathrm{G}$, et al. A conceptual framework for research on subjective cognitive decline in preclinical Alzheimer's disease. Alzheimers Dement 2014;10:844-852.

2. Glodzik-Sobanska L, Reisberg B, De Santi S, Babb JS, Pirraglia $\mathrm{E}$, Rich KE, et al. Subjective memory complaints: presence, severity and future outcome in normal older subjects. Dement Geriatr Cogn Disord 2007;24:177-184.

3. Amariglio RE, Becker JA, Carmasin J, Wadsworth LP, Lorius N, Sullivan C, et al. Subjective cognitive complaints and amyloid burden in cognitively normal older individuals. Neuropsychologia 2012;50:2880-2886.

4. Visser PJ, Verhey F, Knol DL, Scheltens P, Wahlund LO, Freund-Levi Y, et al. Prevalence and prognostic value of CSF markers of Alzheimer's disease pathology in patients with subjective cognitive impairment or mild cognitive impairment in the DESCRIPA study: a prospective cohort study. Lancet Neurol 2009;8:619-627.

5. Saykin AJ, Wishart HA, Rabin LA, Santulli RB, Flashman LA, West JD, et al. Older adults with cognitive complaints show brain atrophy similar to that of amnestic MCI. Neurology 2006;67:834-842.

6. Jessen F, Wiese B, Bachmann C, Eifflaender-Gorfer S, Haller F, Kölsch $\mathrm{H}$, et al. Prediction of dementia by subjective memory impairment: effects of severity and temporal association with cognitive impairment. Arch Gen Psychiatry 2010;67:414-422.

7. Scheef L, Spottke A, Daerr M, Joe A, Striepens N, Kölsch H, et al. Glucose metabolism, gray matter structure, and memory decline in subjective memory impairment. Neurology 2012; 79:1332-1339.

8. Rami L, Fortea J, Bosch B, Solé-Padullés C, Lladó A, Iranzo A, et al. Cerebrospinal fluid biomarkers and memory present dis- 
tinct associations along the continuum from healthy subjects to AD patients. J Alzheimers Dis 2011;23:319-326.

9. Eliassen CF, Reinvang I, Selnes P, Grambaite R, Fladby T, Hessen E. Biomarkers in subtypes of mild cognitive impairment and subjective cognitive decline. Brain Behav 2017;7: e00776.

10. Snitz BE, Weissfeld LA, Cohen AD, Lopez OL, Nebes RD, Aizenstein $\mathrm{HJ}$, et al. Subjective cognitive complaints, personality and brain amyloid-beta in cognitively normal older adults. Am J Geriatr Psychiatry 2015;23:985-993.

11. Amariglio RE, Mormino EC, Pietras AC, Marshall GA, Vannini $\mathrm{P}$, Johnson KA, et al. Subjective cognitive concerns, amyloid- $\beta$, and neurodegeneration in clinically normal elderly. Neurology 2015;85:56-62.

12. Kryscio RJ, Abner EL, Cooper GE, Fardo DW, Jicha GA, Nelson PT, et al. Self-reported memory complaints: implications from a longitudinal cohort with autopsies. Neurology 2014;83:1359-1365.

13. Barnes LL, Schneider JA, Boyle PA, Bienias JL, Bennett DA. Memory complaints are related to Alzheimer disease pathology in older persons. Neurology 2006;67:1581-1585.

14. Vannini P, Hanseeuw B, Munro CE, Amariglio RE, Marshall GA, Rentz DM, et al. Hippocampal hypometabolism in older adults with memory complaints and increased amyloid burden. Neurology 2017;88:1759-1767.

15. Song IU, Choi EK, Oh JK, Chung YA, Chung SW. Alteration patterns of brain glucose metabolism: comparisons of healthy controls, subjective memory impairment and mild cognitive impairment. Acta Radiol 2016;57:90-97.

16. Ryu SY, Lim EY, Na S, Shim YS, Cho JH, Yoon B, et al. Hippocampal and entorhinal structures in subjective memory impairment: a combined MRI volumetric and DTI study. Int Psychogeriatr 2017;29:785-792.

17. Fischl B, Dale AM. Measuring the thickness of the human cerebral cortex from magnetic resonance images. Proc Natl Acad Sci U S A 2000;97:11050-11055.

18. Ashburner J. Computational anatomy with the SPM software. Magn Reson Imaging 2009;27:1163-1174.

19. Lerch JP, Pruessner J, Zijdenbos AP, Collins DL, Teipel SJ, Hampel $\mathrm{H}$, et al. Automated cortical thickness measurements from MRI can accurately separate Alzheimer's patients from normal elderly controls. Neurobiol Aging 2008;29:23-30.

20. Schultz SA, Oh JM, Koscik RL, Dowling NM, Gallagher CL, Carlsson CM, et al. Subjective memory complaints, cortical thinning, and cognitive dysfunction in middle-aged adults at risk for AD. Alzheimers Dement (Amst) 2015;1:33-40.

21. Meiberth D, Scheef L, Wolfsgruber S, Boecker H, Block W, Träber $\mathrm{F}$, et al. Cortical thinning in individuals with subjective memory impairment. J Alzheimers Dis 2015;45:139-146.

22. Cao B, Mwangi B, Passos IC, Wu MJ, Keser Z, Zunta-Soares $\mathrm{GB}$, et al. Lifespan gyrification trajectories of human brain in healthy individuals and patients with major psychiatric disorders. Sci Rep 2017; 7:511.
23. Lebed E, Jacova C, Wang L, Beg MF. Novel surface-smoothing based local gyrification index. IEEE Trans Med Imaging 2013; 32:660-669.

24. Dale AM, Fischl B, Sereno MI. Cortical surface-based analysis. 1. Segmentation and surface reconstruction. Neuroimage 1999;9:179-194.

25. Fischl B, Sereno MI, Dale AM. Cortical surface-based analysis. II: inflation, flattening, and a surface-based coordinate system. Neuroimage 1999;9:195-207.

26. Schaer M, Cuadra MB, Tamarit L, Lazeyras F, Eliez S, Thiran JP. A surface-based approach to quantify local cortical gyrification. IEEE Trans Med Imaging 2008;27:161-170.

27. Williams VJ, Juranek J, Cirino P, Fletcher JM. Cortical thickness and local gyrification in children with developmental dyslexia. Cereb Cortex 2018;28:963-973.

28. Fischl B, Sereno MI, Tootell RB, Dale AM. High-resolution intersubject averaging and a coordinate system for the cortical surface. Hum Brain Mapp 1999;8:272-284.

29. Liu CC, Liu CC, Kanekiyo T, Xu H, Bu G. Apolipoprotein E and Alzheimer disease: risk, mechanisms and therapy. Nat Rev Neurol 2013;9:106-118.

30. Morris JC, Heyman A, Mohs RC, Hughes JP, van Belle G, Fillenbaum $\mathrm{G}$, et al. The consortium to establish a registry for Alzheimer's disease (CERAD). Part I. clinical and neuropsychological assessment of Alzheimer's disease. Neurology 1989;39:1159-1165.

31. Lee DY, Lee KU, Lee JH, Kim KW, Jhoo JH, Kim SY, et al. A normative study of the CERAD neuropsychological assessment battery in the Korean elderly. I Int Neuropsychol Soc 2004; 10:72-81.

32. Folstein MF, Folstein SE, McHugh PR. "Mini-mental state”. A practical method for grading the cognitive state of patients for the clinician. J Psychiatr Res 1975;12:189-198.

33. Lee DY, Lee KU, Lee JH, Kim KW, Jhoo JH, Youn JC, et al. A normative study of the mini-mental state examination in the Korean elderly. J Korean Neuropsychiatr Assoc 2002;41:508525.

34. Jung IK, Kwak DI, Joe SH, Lee HS. A study of standardization of Korean form of Geriatric Depression Scale(KGDS). J Korean Geriatr Psychiatry 1997;1:61-72.

35. Liu T, Wen W, Zhu W, Kochan NA, Trollor JN, Reppermund $\mathrm{S}$, et al. The relationship between cortical sulcal variability and cognitive performance in the elderly. Neuroimage 2011; 56:865-873.

36. Liu T, Lipnicki DM, Zhu W, Tao D, Zhang C, Cui Y, et al. Cortical gyrification and sulcal spans in early stage Alzheimer's disease. PLoS One 2012;7:e31083.

37. Bogousslavsky J, Miklossy J, Deruaz JP, Assal G, Regli F. Lingual and fusiform gyri in visual processing: a clinicopathologic study of superior altitudinal hemianopia. J Neurol Neurosurg Psychiatry 1987; 50:607-614.

38. Slotnick SD. Visual memory and visual perception recruit common neural substrates. Behav Cogn Neurosci Rev 2004; 
3:207-221.

39. Miller BL, Boeve BF. The behavioral neurology of dementia. Cambridge:Cambridge University Press,2009.

40. Jeong HS, Park JS, Song IU, Chung YA, Rhie SJ. Changes in cognitive function and brain glucose metabolism in elderly women with subjective memory impairment: a 24-month prospective pilot study. Acta Neurol Scand 2017;135:108-114.

41. Chételat G, Desgranges B, De La Sayette V, Viader F, Eustache F, Baron JC. Mapping gray matter loss with voxel-based morphometry in mild cognitive impairment. Neuroreport 2002;13:1939-1943.

42. Chételat G, Landeau B, Eustache F, Mézenge F, Viader F, de la Sayette $\mathrm{V}$, et al. Using voxel-based morphometry to map the structural changes associated with rapid conversion in MCl: a longitudinal MRI study. Neuroimage 2005;27:934-946.

43. Yetkin FZ, Rosenberg RN, Weiner MF, Purdy PD, Cullum CM. FMRI of working memory in patients with mild cognitive impairment and probable Alzheimer's disease. Eur Radiol 2006; 16:193-206.

44. Rodrigue KM, Raz N. Shrinkage of the entorhinal cortex over five years predicts memory performance in healthy adults. J Neurosci 2004;24:956-963.

45. Minoshima S, Giordani B, Berent S, Frey KA, Foster NL, Kuhl DE. Metabolic reduction in the posterior cingulate cortex in very early Alzheimer's disease. Ann Neurol 1997;42:85-94.

46. Liu Y, Paajanen T, Zhang Y, Westman E, Wahlund LO, Simmons A, et al. Analysis of regional MRI volumes and thicknesses as predictors of conversion from mild cognitive impairment to Alzheimer's disease. Neurobiol Aging 2010;31: 1375-1385.

47. Hu S, Ide JS, Zhang S, Li CR. The right superior frontal gyrus and individual variation in proactive control of impulsive response. J Neurosci 2016;36:12688-12696.

48. Michalski LJ, Demers CH, Baranger DAA, Barch DM, Harms MP, Burgess GC, et al. Perceived stress is associated with in creased rostral middle frontal gyrus cortical thickness: a family-based and discordant-sibling investigation. Genes Brain Behav 2017;16:781-789.

49. Weston PS, Nicholas JM, Lehmann M, Ryan NS, Liang Y, Macpherson $\mathrm{K}$, et al. Presymptomatic cortical thinning in familial Alzheimer disease: a longitudinal MRI study. Neurology
2016;87:2050-2057.

50. Blanc F, Colloby SJ, Cretin B, de Sousa PL, Demuynck C, O'Brien JT, et al. Grey matter atrophy in prodromal stage of dementia with Lewy bodies and Alzheimer's disease. Alzheimers Res Ther 2016;8:31.

51. Ferraro FR. Aging, Alzheimer's disease, and word recognition: a review of the recent literature. In: Allen PA, Bashore TR, editors. Age differences in word and language processing. Amsterdam:Elsevier; 1995. p.220-246.

52. Cai $\mathrm{K}, \mathrm{Xu} \mathrm{H}$, Guan $\mathrm{H}$, Zhu W, Jiang J, Cui Y, et al. Identification of early-stage Alzheimer's disease using sulcal morphology and other common neuroimaging indices. PLoS One 2017; 12:e0170875.

53. Lau WK, Leung MK, Lee TM, Law AC. Resting-state abnorma/ities in amnestic mild cognitive impairment: a meta-analysis. Transl Psychiatry 2016;6:e790.

54. Redolfi A, Manset D, Barkhof F, Wahlund LO, Glatard T, Mangin JF, et al. Head-to-head comparison of two popular cortical thickness extraction algorithms: a cross-sectional and longitudinal study. PLoS One 2015;10:e0117692.

55. Oh A, Duerden EG, Pang EW. The role of the insula in speech and language processing. Brain Lang 2014;135:96-103.

56. Davatzikos C, Bhatt P, Shaw LM, Batmanghelich KN, Trojanowski JQ. Prediction of MCI to AD conversion, via MRI, CSF biomarkers, and pattern classification. Neurobiol Aging 2011;32:2322.e19-2322.e27.

57. Eskildsen SF, Coupé P, García-Lorenzo D, Fonov V, Pruessner JC, Collins DL. Prediction of Alzheimer's disease in subjects with mild cognitive impairment from the ADNI cohort using patterns of cortical thinning. Neuroimage 2013;65:511-521.

58. Ugur HC, Kahilogullari G, Coscarella E, Unlu A, Tekdemir I, Morcos JJ, et al. Arterial vascularization of primary motor cortex (precentral gyrus). Surg Neurol 2005;64 Suppl 2:S48-S52.

59. Taylor L, Jones L. Effects of lesions invading the postcentral gyrus on somatosensory thresholds on the face. Neuropsychologia 1997;35:953-961.

60. Zlatar ZZ, Moore RC, Palmer BW, Thompson WK, Jeste DV. Cognitive complaints correlate with depression rather than concurrent objective cognitive impairment in the successful aging evaluation baseline sample. J Geriatr Psychiatry Neurol 2014;27:181-187. 